\title{
Motor neurons derived from ALS-related mouse iPS cells recapitulate pathological features of ALS
}

\author{
Ju-Hwang Park ${ }^{1}$, Hang-Soo Park², Sunghoi Hong ${ }^{2}$ and Seongman Kang ${ }^{1}$
}

Amyotrophic lateral sclerosis (ALS) is a late-onset progressive neurodegenerative disease characterized by the loss of motor neurons in the spinal cord and brain. Mutations in $\mathrm{Cu} / \mathrm{Zn}$ superoxide dismutase 1 (SOD1) are known to induce ALS. Although many research models have been developed, the exact pathological mechanism of ALS remains unknown. The recently developed induced pluripotent stem (iPS) cell technology is expected to illuminate the pathological mechanisms and new means of treatment for neurodegenerative diseases. To determine the pathological mechanism of ALS, we generated mouse iPS (miPS) cells from experimental ALS transgenic mice and control mice and characterized the cells using molecular biological methods. The generated miPS cells expressed many pluripotent genes and differentiated into three germ layers in vitro and in vivo. Motor neurons derived from ALS-related miPS cells recapitulated the pathological features of ALS. The ALS-model motor neurons showed SOD1 aggregates, as well as decreased cell survival rate and neurite length compared with wild-type motor neurons. Our study will be helpful in revealing the mechanism of motor neuronal cell death in ALS.

Experimental \& Molecular Medicine (2016) 48, e276; doi:10.1038/emm.2016.113; published online 9 December 2016

\section{INTRODUCTION}

Amyotrophic lateral sclerosis (ALS) is a late-onset neurodegenerative disease characterized by the loss of motor neurons in the spinal cord and brain. ${ }^{1}$ Progressive paralysis of voluntary muscles and progressive spread of symptoms are typical features of ALS. Respiratory failure with denervation of the respiratory muscles and diaphragm is the last symptom in ALS. Most cases of ALS ( $90 \%)$ are sporadic, and the remaining cases $(\sim 10 \%)$ are familial. ${ }^{2}$ Mutations of $\mathrm{Cu} / \mathrm{Zn}$ superoxide dismutase 1 (SOD1) are related to the development of $\sim 20 \%$ of familial ALS cases. ${ }^{3}$ SOD1 is a predominantly cytoplasmic protein that consists of 153 amino acids. SOD1 converts superoxide anion to hydrogen peroxide to protect cells. It was reported that while SOD1 null mice do not develop motor neuron death, mutant SOD1 transgenic mice recapitulate ALS symptoms. ${ }^{4}$ It is thought that mutant SOD1 induces cell death by a gain of function, although the precise pathologic mechanism remains unknown.

There are many theories about the cause of motor neuronal cell death in ALS. These include genetic factors, ${ }^{5}$ oxidative stress, ${ }^{6}$ mitochondrial dysfunction, ${ }^{7}$ ER stress, ${ }^{8}$ excitotoxicity, ${ }^{9}$ proteasome inhibition, ${ }^{10}$ axonal transport defeat, ${ }^{11}$ dysregulation of RNA processing ${ }^{12}$ and formation of protein aggregates. ${ }^{13}$
ALS transgenic mice carrying the human mutant SOD1 (G93A) gene provide a common research model for ALS. ${ }^{14}$ These mice present a pathology similar to that of human ALS patients, such as motor neuronal loss in the brain and spinal cord, the presence of aggregates, inflammation and death. ${ }^{15}$ In particular, these mice present hind limb weakness and tremor around postnatal day 90 and then die at approximately postnatal day 120. Degenerative processes in the motor neurons are observed in the early stages of the development of symptoms, and degeneration of neuromuscular junctions may precede the loss of motor neurons. Pathological characteristics, such as mitochondrial vacuolization, Golgi fragmentation or neurofilament-positive inclusions, are present in the motor neurons of ALS transgenic mice. Motor neurons of these mice are also affected by inflammation that causes astrocytosis and microgliosis.

Recently, somatic reprogramming technology was used to produce induced pluripotent stem (iPS) cells by applying four pluripotent genes, namely, Oct4, Sox2, Klf4 and c-Myc. ${ }^{16}$ Researchers discovered these key pluripotent genes using differentiated mouse embryonic fibroblasts and tested the expression of these genes and the differentiation ability of iPS cells. There are many advantages to using iPS cells. For

\footnotetext{
${ }^{1}$ Division of Life Sciences, College of Life Sciences and Biotechnology, Korea University, Seoul, Republic of Korea and ${ }^{2}$ Department of Integrated Biomedical and Life Science, College of Health Science, Korea University, Seoul, Republic of Korea

Correspondence: Professor S Kang, Division of Life Sciences, College of Life Sciences and Biotechnology, Korea University, 1, 5-ka, Anam-dong, Sungbukgu, Seoul 02841, Republic of Korea.

E-mail: skang@korea.ac.kr

Received 7 March 2016; revised 6 July 2016; accepted 1 August 2016
} 
example, they are easy to create, can be applied in patientspecific cell therapy and research, and require no special ethical considerations. In particular, iPS cells are expected to help identify drugs for the treatment of patients with neurodegenerative disease. ${ }^{17}$ For these reasons, many iPS cell lines have been produced, using human or animal models, for research on ALS. ${ }^{18,19}$

In the present study, we report pathological differences between iPS cell-derived motor neurons from ALS mice and those from control mice; these differences include neural dendrites, aggregates and cell death. Our results demonstrate that motor neurons derived from ALS-related mouse iPS cells recapitulate the pathological features of ALS.

\section{MATERIALS AND METHODS}

\section{Animals}

ALS transgenic mice expressing the human mutant SOD1 (G93A) gene (B6SJL-Tg[SOD1-G93A]1Gur/J) and their non-transgenic littermates (B6SJLF1/J) - the latter used as controls-were purchased from the Jackson Laboratory (Bar Harbor, ME, USA). All mouse care and experiments were agreed upon by the Institutional Animal Care and Use Committee of Korea University.

\section{Tail-tip fibroblast culture from mouse}

Tail-tip fibroblasts (TTFs) were prepared from the transgenic and control mice as previously described. ${ }^{20}$ The TTFs were maintained in Dulbecco's modified minimal essential medium (DMEM; Gibco, Life Technologies, Grand Island, NY, USA) supplemented with 10\% FBS (Gibco).

\section{Retroviral production and titration}

Retroviral production and titration were conducted as described elsewhere. ${ }^{21}$

\section{Generation of mouse iPS cells}

To generate the mouse iPS cells, $5 \times 10^{4}$ mouse TTFs were seeded on a six-well tissue culture plate, and retroviral infection was performed for 3 days on the mouse TTFs with polybrene $\left(4 \mu \mathrm{g} \mathrm{ml}^{-1}\right)$. After 3 days, the infected TTFs were transferred to Mouse Embryonic Fibroblast (MEF) feeder cells to produce mouse iPS cells with mouse iPS medium (DMEM containing 10\% horse serum (Sigma-Aldrich, St Louis, MO, USA), 2 mM L-glutamine (Gibco), $0.1 \mathrm{~mm} \mathrm{MEM} \mathrm{NEAA}$ (Gibco), 10 mm HEPES (Gibco), 10 mm $\beta$-mercaptoethanol (Gibco), $500 \mathrm{U} \mathrm{ml}^{-1}$ LIF (Millipore, Billerica, MA, USA) and penicillin/ streptomycin (Gibco)).

\section{Alkaline phosphatase staining}

For the alkaline phosphatase staining, we used the Alkaline Phosphatase Staining Kit (Stemgent, Lexington, MA, USA) according to the manufacturer's protocol.

\section{RT-PCR}

Total RNA was extracted from the established mouse iPS cells with the easy-Blue Total RNA Extraction Kit (Intron, Seongnam, Korea) and then reverse-transcribed into first-strand cDNA using the RevertAid $\mathrm{H}$ Minus First Strand cDNA Synthesis Kit (Fermentas, Thermo Fischer Scientific, Carlsbad, CA, USA). The primers against each of the pluripotent genes were described. ${ }^{20}$

\section{Immunofluorescence}

The mouse iPS colonies were fixed with $4 \%$ formaldehyde for $15 \mathrm{~min}$ and permeabilized with $0.1 \%$ Triton X-100. After incubation with $2 \%$ $\mathrm{BSA}$, the cells were incubated overnight at $4{ }^{\circ} \mathrm{C}$ with a primary antibody against Oct4 (Abcam, Cambridge, MA, USA), Sox2 (Millipore), Nanog (Millipore) and SSEA-1 (Santa Cruz, Dallas, TX, USA). The cells were then washed three times in phosphate-buffered saline and incubated with Alexa Fluor 488 and 594 (Life Technologies, Thermo Fisher Scientific, Carlsbad, CA, USA) for $1 \mathrm{~h}$ at room temperature. After washing, the nuclei were stained with DAPI (Sigma-Aldrich). The stained mouse iPS colonies were mounted on glass slides using Fluorescent Mounting Medium (Dako, Agilent Technologies, Denmark). The fluorescent images were captured using a LSM-700 confocal microscope (Carl Zeiss, Oberkochen, Germany).

\section{In vitro differentiation}

Mouse iPS cells were directly differentiated into three germ layers on a gelatin-coated dish for 10 days in a medium without a leukemia inhibitory factor.

\section{Teratoma formation}

For the teratoma formation, we harvested mouse iPS cells and subcutaneously injected $1 \times 10^{6}$ mouse iPS cells into SCID mice. After 6 weeks, the formed teratomas were analyzed by hematoxylin and eosin (H\&E) staining.

\section{Bisulfite genomic DNA sequencing}

Genomic DNA was isolated from mouse embryonic stem cells (mESs), MEFs and miPS cells using the QIAmp DNA Mini Kit (Qiagen, Valencia, CA, USA) for bisulfite modification. Bisulfite modification was performed using a CpGenome Modification Kit (Millipore) according to the manufacturer's protocol. Modified genomic DNA was amplified by PCR using a mouse Nanog gene promoter primer. ${ }^{16}$ The amplified PCR products were cloned into a T\&A Cloning Vector Kit (RBC, New Taipei City, Taiwan) and sequenced using the BIQ Analyzer software (Max Planck Institute, Saarbrucken, Germany).

\section{Motor neuron differentiation of miPS cells}

For the motor neuron differentiation, a procedure modified from a previous study was used. ${ }^{22}$ First, embryoid bodies (EBs) were formed in a suspension culture for 4 days using an EB medium (DMEM containing 10\% FBS (Gibco), 2 mm L-glutamine (Gibco), $0.1 \mathrm{~mm}$ MEM NEAA (Gibco), $10 \mathrm{~mm}$ HEPES (Gibco), $10 \mathrm{~mm}$ $\beta$-mercaptoethanol (Gibco) and penicillin/streptomycin (Gibco)). After 4 days, the EBs were cultured for $\sim 10$ days in a tissue culture dish using an insulin-transferrin-selenium (ITS) medium (DMEM/F12 (Gibco) containing ITS supplement (Gibco) and penicillin/streptomycin (Gibco)) to select neural precursor cells. The ITS medium was replaced every 2 or 3 days. To promote the proliferation of neural precursor cells, selected neural precursor cells were detached by Trypsin-EDTA and maintained in an N2 medium (DMEM/F12 (Gibco) containing N2 supplement (Gibco) and penicillin/streptomycin (Gibco)) with bFGF (10 $\left.\mathrm{ng} \mathrm{ml}^{-1}\right)$. Enriched neural precursor cells were differentiated into motor neurons using retinoic acid $(1 \mu \mathrm{M})$ and Shh (200 ng ml ${ }^{-1}$ ) in an N2 medium. After differentiation, the motor neurons were cultured in an N2 medium containing ascorbic acid for their maturation and survival. 
TUNEL assay

For the terminal deoxynucleotidyl transferase dUTP nick end labeling (TUNEL) assay, the DeadEnd Fluorometric TUNEL System Kit (Promega, Madison, WI, USA) was used according to the manufacturer's protocol.

\section{Measurement of neurite length}

To measure the neurites of differentiated motor neurons, differentiated motor neurons were immunostained with Tuj1 and Islet-1 antibodies. As Tuj1 was expressed in the cytosol, we measured neurite length by immunostaining Tuj1 from cell body to end of neurite in co-immunostained motor neurons. The length of the neurite was calculated using the measurement function (using 'Open Bezier') of the ZEN analysis program (Carl Zeiss, Oberkochen, Germany).

\section{Statistical analysis}

The differences between the various experimental groups were calculated using Student's two-tailed $t$-test. $P$-values of $<0.05$ were considered to be statistically significant.

\section{RESULTS}

\section{Establishment of miPS cells from ALS-related transgenic mice TTFs}

We produced mouse iPS cells from primary mouse TTFs derived from ALS transgenic mice and control mice. First, we obtained fibroblasts from mouse tail-tip tissue and cultured them for 2 weeks for sufficient viral infection (Figure 1b). We reprogrammed the mouse TTFs by infection with four pluripotent genes (Oct4, Sox2, Klf4 and c-Myc) using a retrovirus for 3 days. We then seeded the infected TTFs with MEF feeder cells to maintain the generation of miPS cells. After 6 days, the first miPS colony was observed in the infected ALS-TTFs (Figure 1a). On day 15 (D15), we picked multiple miPS colonies and transferred them to other MEF feeder cells (Figure 1c). Next, miPS colonies were counted during D14 to D22 to measure the differences between WT-iPS and ALS-iPS cell lines. Although there were a few differences in the total number of colonies on a single day, the rate of increase between WT- and ALS-iPS cells was almost equal during the counting period (WT-iPS; 18 and ALS-iPS; 15 colonies per day) (Figure 1d). Thus, we can conclude that the mutant SOD1 gene did not affect the production of miPS cells.

\section{Established miPS cells from ALS-model mice had pluripotent properties}

Many molecular biological experiments were conducted to characterize the established WT-iPS and ALS-iPS cell lines. To evaluate the iPS properties of these established miPS cells, we investigated alkaline phosphatase activity (Figure 2a). Alkaline phosphatase staining experiments revealed that the WT-iPS and ALS-iPS cell lines were positive for alkaline phosphatase activity. Next, we analyzed the mRNA expression of pluripotent genes, such as Ecat1, Nanog, Gdf3, Oct4, Sox2, Rex1 and Zfp296 by RT-PCR (Figure 2b). All cell lines of WT-iPS and ALS-iPS expressed these genes. Moreover, all cell lines showed immunostaining for pluripotent markers, such as Oct4, Sox2, Nanog and SSEA-1 (Figures 2c and d).
To test the in vitro-differentiation ability of the established miPS cells, WT-iPS and ALS-iPS were differentiated in a LIF-free medium. The differentiated WT-iPS and ALS-iPS cells were immunostained with Tuj-1 (ectoderm), AFP (endoderm) and Desmin (mesoderm) antibodies. The results demonstrate that the established miPS cells differentiated to three germ layers in vitro (Figure 2e).

Furthermore, we also tested the in vivo-differentiation potential of the established miPS cells by observing teratoma formation. Teratomas formed within 6 weeks of the injection of the miPS cells. The teratomas were analyzed by $\mathrm{H} \& \mathrm{E}$ staining (Figure 2f). The H\&E staining revealed that the teratomas included three germ layers: epidermis and neural tissue (ectoderm), muscle and blood (mesoderm), and intestine and gland (endoderm). These results demonstrate that the established miPS cells have the potential to differentiate into three germ layers in vivo.

Finally, we investigated epigenetic changes in the Nanog promoter in the established miPS cells (Figure 2g). Bisulfite sequencing analysis showed that the methylation patterns of WT-iPS and ALS-iPS were similar to the mES cell pattern.

Taken together, the results demonstrate that the established miPS had properties of general iPS cells and that mutant SOD1 gene did not affect pluripotency, differentiation ability and epigenetic change.

\section{ALS-iPS cells differentiated into motor neurons in vitro}

Because the features of ALS were confirmed in motor neurons, we differentiated WT-iPS and ALS-iPS cells into motor neurons using the five-stage differentiation method with minor modifications (Figure 3a). First, we induced EB formation to differentiate the miPS cells without LIF for 4 days. Well-shaped EBs were observed in the culture dish with ITS medium for 10 days. Selected neural precursor cells were cultured with bFGF to promote the proliferation of neural precursor cells. Finally, enriched neural precursor cells were differentiated into motor neurons with RA and Shh. We monitored all of the differentiation steps by observing the differentiating cells (Figure 3b). To test the differentiation of the motor neurons, Islet-1 and Tuj-1 immunostaining experiments were performed. Figure $3 c$ shows successfully differentiated motor neurons. Moreover, other motor neuron markers, such as HB9 and ChAT, were also observed in the differentiated motor neurons (Figure 3d). We measured the Islet-1 positive/Tuj-1 positive cells to evaluate the differentiation rate of neural precursor cells into motor neurons. Approximately 86.1 and $87.3 \%$ of the neural precursor cells differentiated into motor neurons among the WT- and ALS-iPS cells, respectively (Figure 3e). Therefore, we can conclude that there was no difference in the motor neuron differentiation between WT- and ALS-iPS cells and that the mutant SOD1 gene does not affect motor neuron differentiation.

\section{ALS-motor neurons recapitulate pathological features of ALS} Considering that a decrease in motor neuron neurite length is one of the major characteristics of ALS pathology, we evaluated 


\section{a}

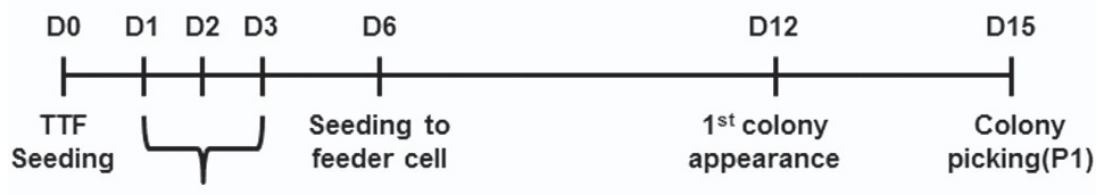

Viral infection

(Oct4, Sox2, KIf4, c-Myc)

b

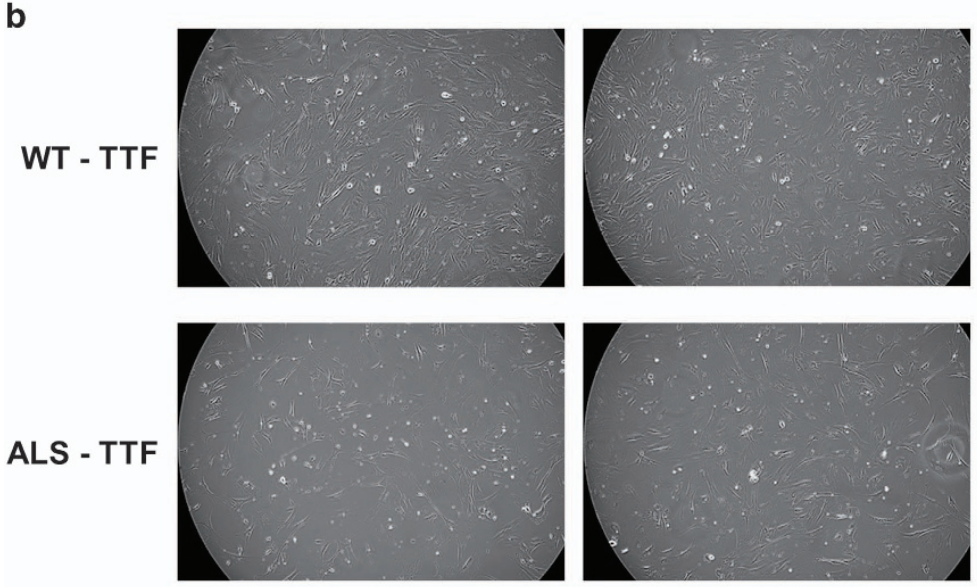

c
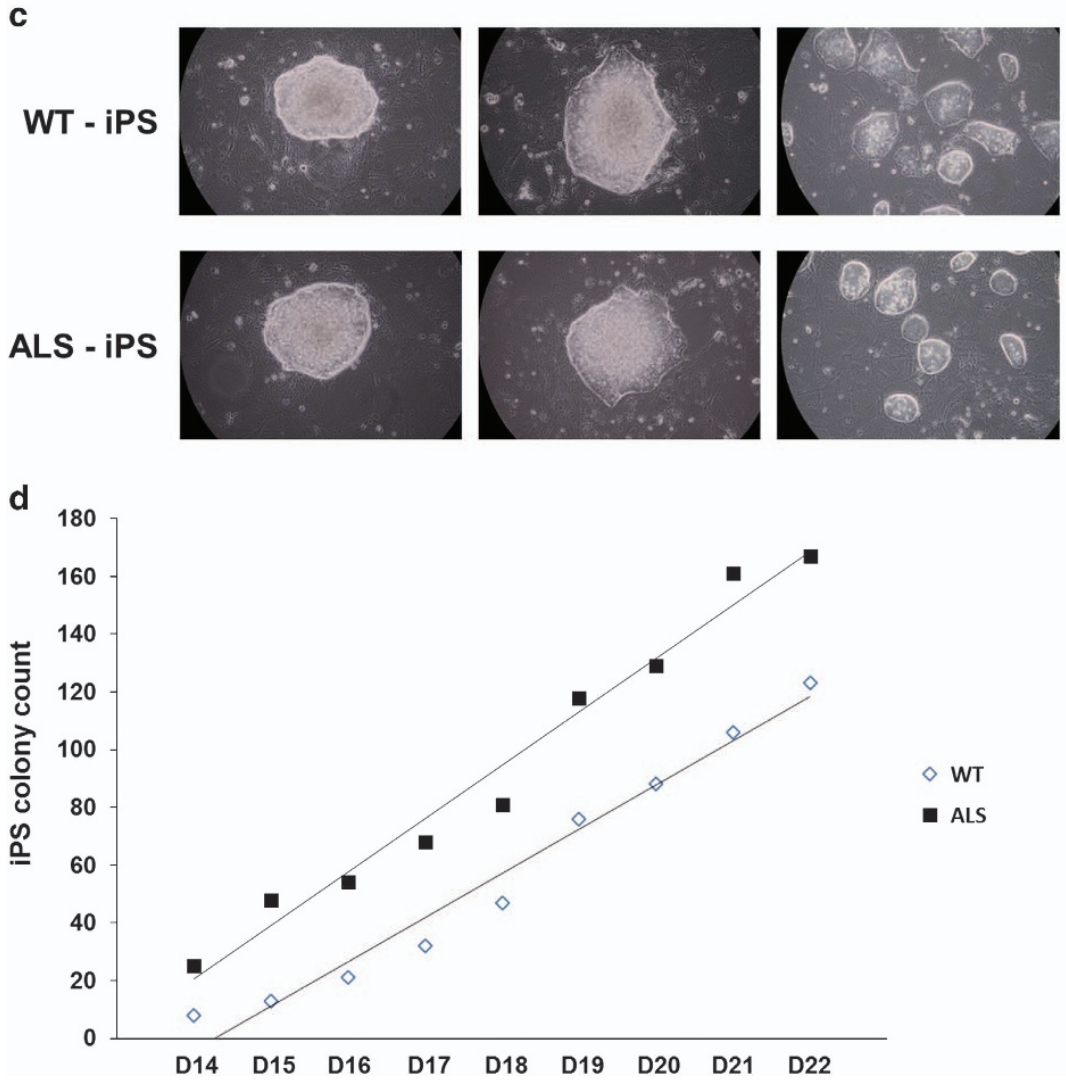

$\diamond W T$

- ALS

Figure 1 Production of miPS cells from ALS-related transgenic mice using pluripotent genes. (a) A schematic of the construction of mouse iPS (miPS) cells. To produce miPS cells, TTFs were infected with a retrovirus containing four pluripotent genes (Oct4, Sox2, KIf4 and c-Myc). (b) Primary fibroblasts derived from ALS-related transgenic mice and control mice tail tips. To obtain a sufficient number of cells for viral infection, we cultured cells for 2 weeks. (c) The WT-iPS and ALS-iPS colonies generated on the feeder cells were round and hyaloid in shape and appearance. (d) The graph shows that the production rate of the miPS colony counted from D14 to D22 was similar between WT-iPS and ALS-iPS. 
a

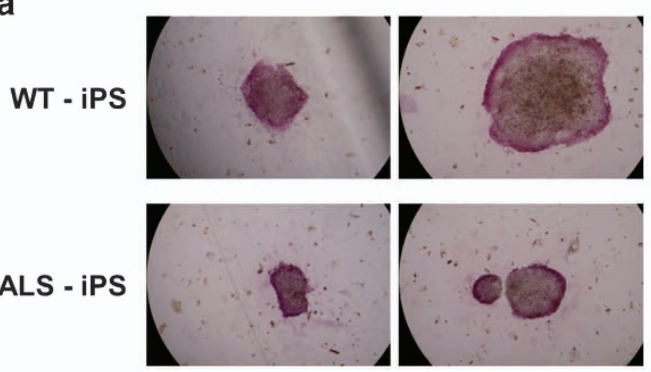

C

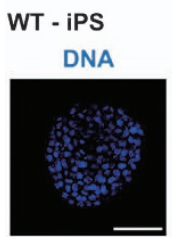

DNA

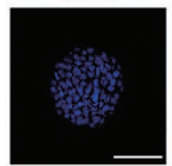

e

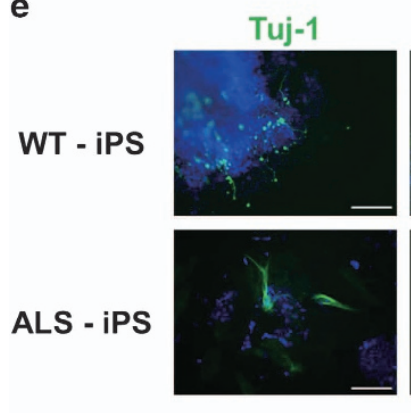

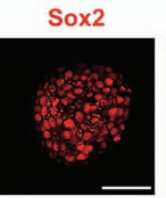

SSEA-1

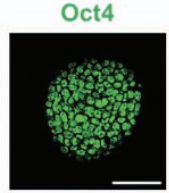

Nanog

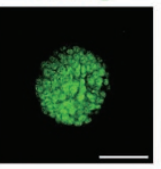

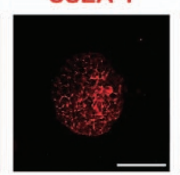

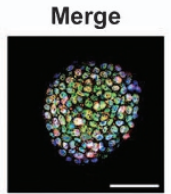

Merge

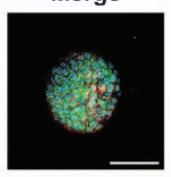

b

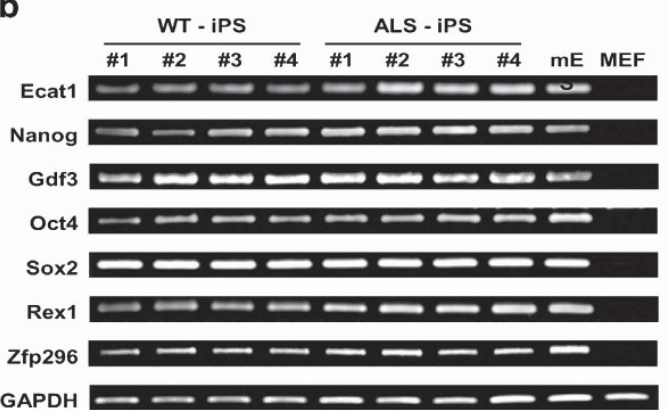

d

ALS - iPS

DNA

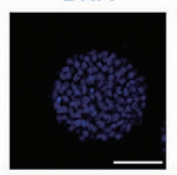

DNA

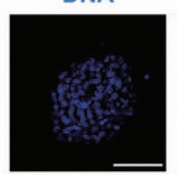

Nanog

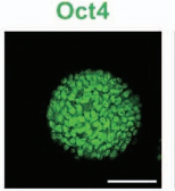

Sox2
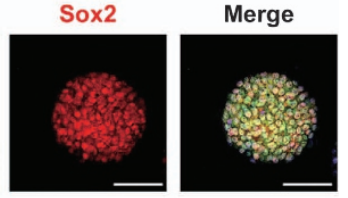

SSEA-1
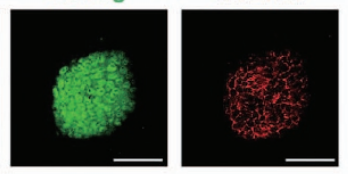

Merge

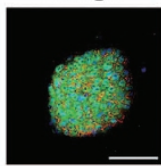

g
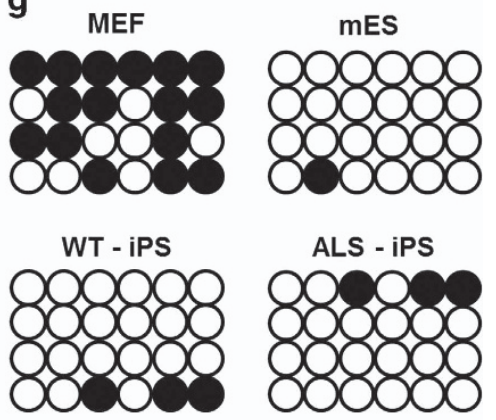

f

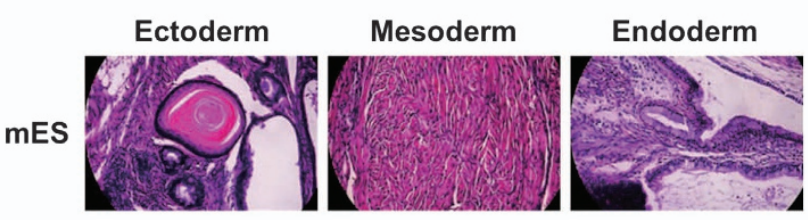

WT-iPS
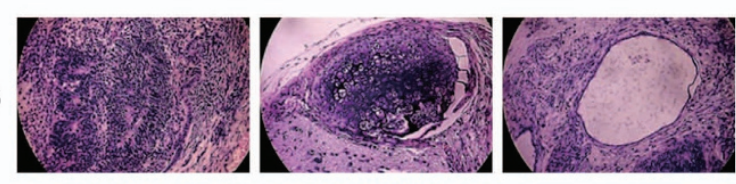

ALS-iPS
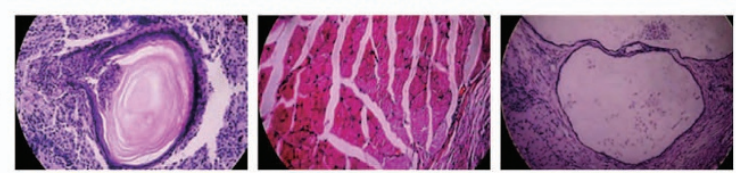

Figure 2 Characterization of miPS cells derived from ALS-related mice. (a) WT-iPS and ALS-iPS cells were positive for alkaline phosphatase staining. (b) RT-PCR shows that all WT-iPS and ALS-iPS cell lines expressed pluripotent genes. (c) WT-iPS cells were immunostained with Oct4 (green), Sox2 (red), Nanog (green) and SSEA-1 (red). Scale bar, $100 \mu$ m. (d) ALS-iPS cells were immunostained with Oct4 (green), Sox2 (red), Nanog (green) and SSEA-1 (red). Scale bar, $100 \mu \mathrm{m}$. (e) The in vitro differentiation experiment showed that WT-iPS and ALS-iPS cells randomly differentiated into three germ layers. Scale bar, $200 \mu \mathrm{m}$. (f) Teratoma formation showed that WT-iPS and ALS-iPS differentiated into three germ layers of tissues in vivo. (g) Nanog promoter methylation patterns of WT-iPS and ALS-iPS cells were similar to mES patterns but not to MEF. 
a

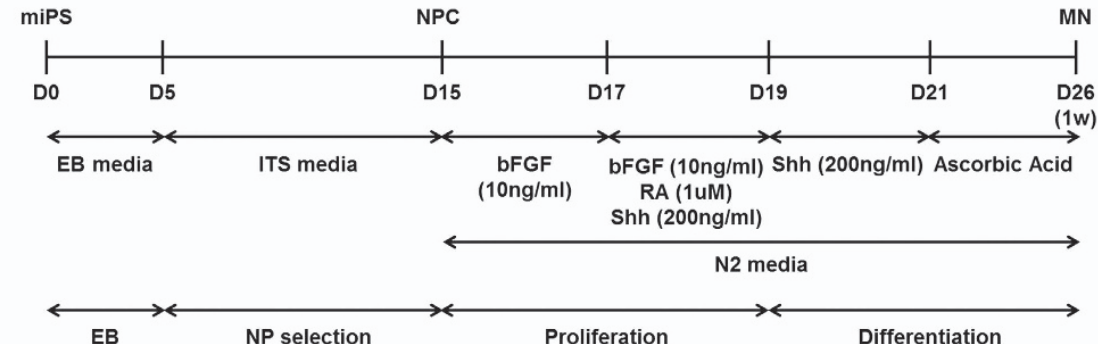

b

EB (D4)

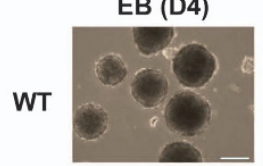

ALS
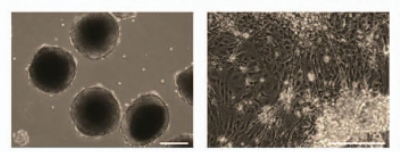

d

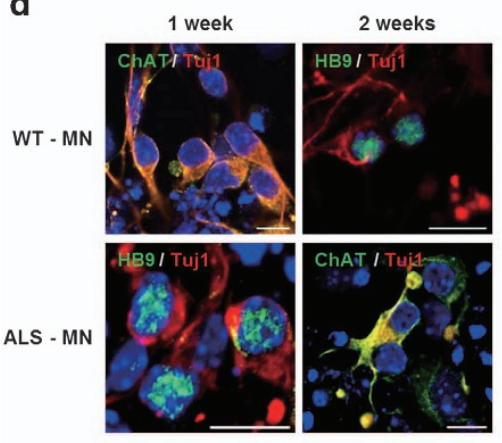

c
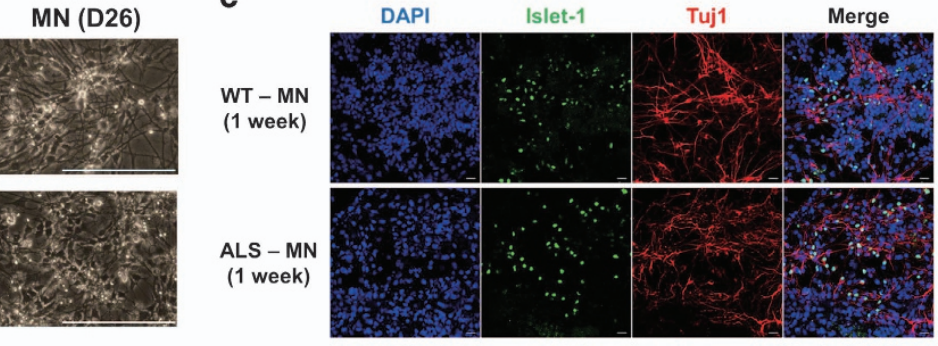

e

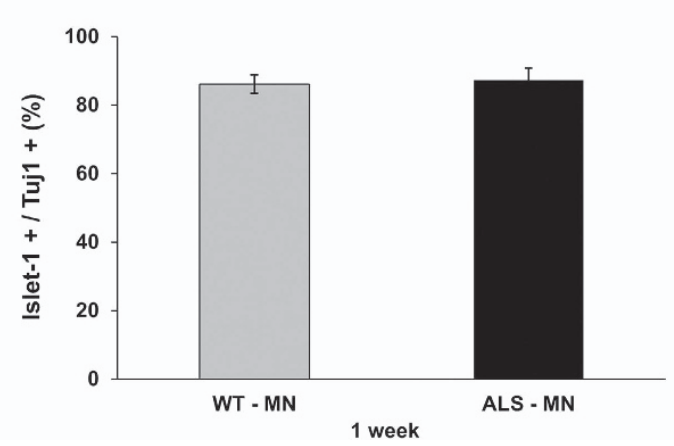

Figure 3 Motor neuron differentiation of ALS-iPS by a five-stage method. (a) A schematic diagram showing motor neuron differentiation of the mouse iPS cells. To differentiate WT-iPS and ALS-iPS cells into EB, Neural Precursor Cell (NPC) and MN stages, the established miPS cells were treated with different media. (b) Morphology of each stage of motor neuron differentiation. Scale bar, $200 \mu \mathrm{m}$. (c) Differentiation of motor neurons from WT-iPS and ALS-iPS cells was examined by Islet-1 (green) and Tuj-1 (red) double immunostaining. Tuj-1 is a pan neuronal marker and Islet-1 is a motor neuron marker. Scale bar, $20 \mu \mathrm{m}$. (d) Differentiation of motor neurons from WT-iPS and ALS-iPS was confirmed by HB9 (green) and ChAT (green). Scale bar, $10 \mu \mathrm{m}$. (e) The graph shows differentiation rates from neuronal precursors to motor neurons. The results reflect five independent experiments. Error bar reflects s.d.

the motor neuron neurite lengths of induced WT- and ALS-MNs. We immunostained the cells with Islet-1 and Tuj-1 antibodies to distinguish motor neurons from other cells. To estimate the differences in neurite length between the induced WT- and ALS-MNs, we selected Islet-1 and Tuj-1 double-positive cells and measured neurite lengths using the Tuj-1 immunostaining (Figure 4a). A week after differentiation, the relative neurite length of ALS-MNs (0.68) decreased more than the neurites of WT-MNs (1) (Figure 4b). Moreover, 3 weeks after differentiation, the relative neurite length of ALS-MNs (0.56) decreased to a greater extent (Figure 4c). These results demonstrate that relative neurite lengths of ALS-motor neurons continuously decrease in the process of motor neuron maturation.

The mutant SOD1 aggregate is the principal pathological feature of ALS. Intracellular SOD1 aggregates were observed in ALS-MNs (Figure 4d), showing that the mutant SOD1 gene affects the physiology of the motor neuron state. Taken together, these results let us conclude that the induced ALS-motor neurons recapitulate the pathological features of ALS.

\section{Cell survival of ALS motor neurons is decreased}

The most important characteristic of ALS is death of motor neurons. We investigated motor neuron death using the TUNEL assay. We counted cells that were double positive for Islet-1 and TUNEL to distinguish motor neuron cells and determine motor neuronal death (Figure 5a). The results showed that the cell survival rate of ALS-MNs was $~ 47.9 \%$ compared with that of WT-MNs (Figure 5b). Furthermore, we assessed cell death in the iPS stage using the TUNEL assay (Figure 5c). There were 3.4 and 4.1 dead cells per iPS colony in the WT- and ALS-iPS colonies, respectively; thus, WT- and ALS-iPS colonies appeared to have similar proportions of cell 
a
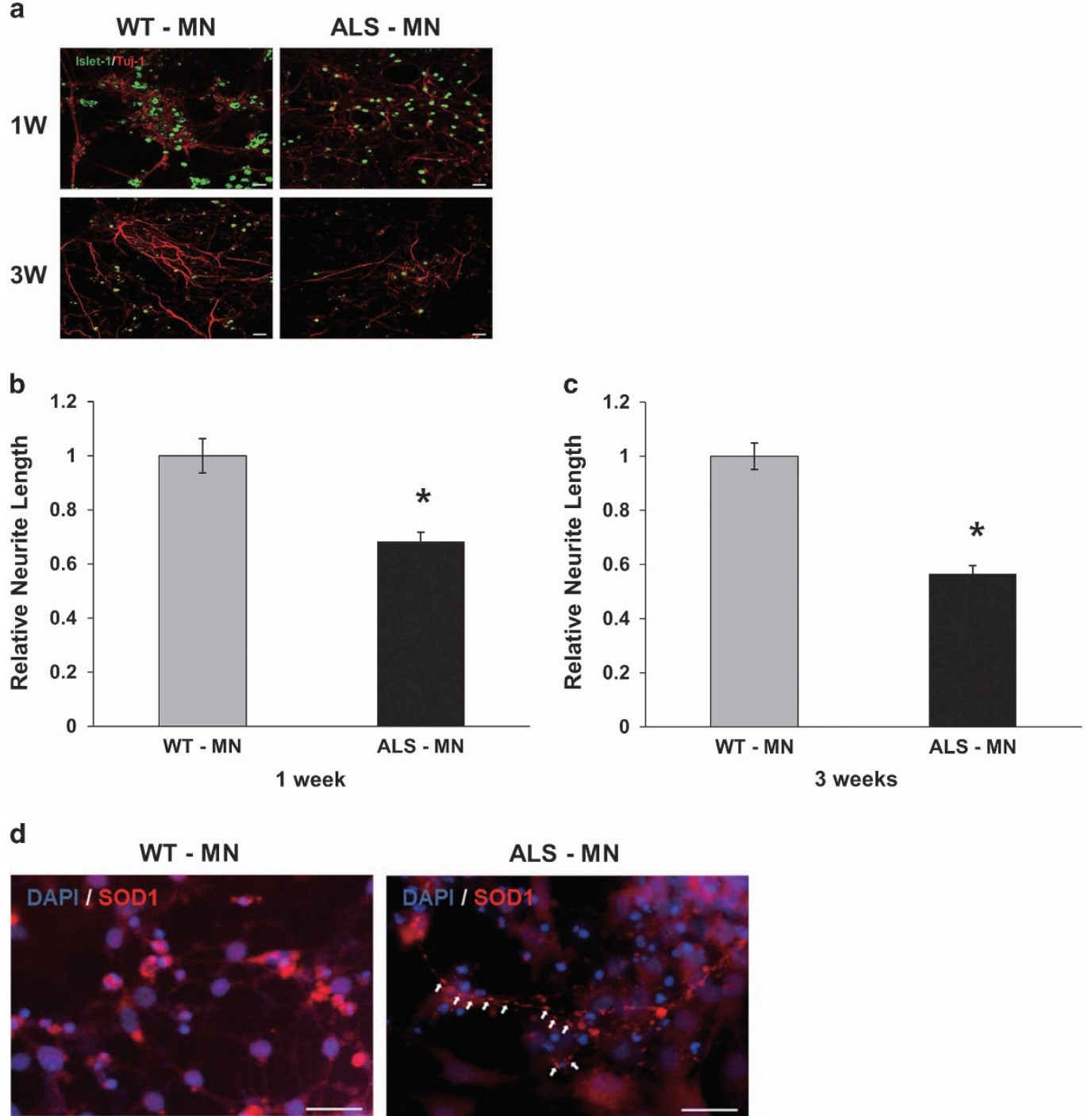

Figure 4 ALS-motor neurons show distinct characteristics of ALS. (a) Immunostaining of differentiated WT-MNs and ALS-MNs. Islet-1 (green) is a motor neuron marker and Tuj-1 (red) is a pan neuronal marker. Scale bar, $20 \mu \mathrm{m}$. (b) Relative neurite length of ALS-MNs (0.68) was shorter than WT-MNs (1) at 1 week after differentiation $(P<0.005)$. $(n=20$ (WT), 22 (ALS)) Error bar reflects s.d. (c) Relative neurite length of ALS-MNs (0.56) was shorter than WT-MNs (1) at 3 weeks after differentiation $(P<0.001)$. $(n=25$ (WT), 14 (ALS)) Error bar reflects s.d. (d) White arrows in immunostained ALS-MNs show mutant SOD1 aggregates in motor neurons. Scale bar, $200 \mu \mathrm{m}$

death (Figure 5d). Thus, we can conclude that induced ALS-MNs and WT-MNs did not show any difference of cell death rate in the embryonic stage, but showed a drastic difference in the motor neuron stage.

\section{DISCUSSION}

The pathological mechanism and medical treatment of many neurodegenerative diseases, including ALS, are uncertain. Because neurodegenerative diseases have a late onset, the investigation of their causes and processes is very difficult. Although many animal models have been developed, there is no system for studying the development of ALS from the embryonic to the adult stage. The use of iPS cells has many advantages in neurodegenerative disease research, such as avoiding ethical considerations and providing patient-specific therapy. ${ }^{17}$ In the present study, we used iPS cells derived from a transgenic mouse model to reveal the pathological mechanism of ALS. The results of our study will be helpful in revealing the mechanism of motor neuronal cell death in ALS.

We produced mouse iPS cells using TTFs derived from ALS transgenic mice (Figure 1). The generated miPS cells developed a rounded and brightened appearance on the MEF feeder cells. In particular, we investigated the number of miPS colonies produced after viral infection to reveal the relationship between the transduced mutant SOD1 gene and miPS-cell production. The results demonstrate that overexpressed mutant SOD1 did not affect the production of the miPS cells.

Moreover, the characterization of the miPS cells suggests that overexpressed mutant SOD1 does not affect the miPS cells. No differences were found between WT-iPS and ALS-iPS cells in alkaline phosphatase staining, RT-PCR, immunostaining, in vitro differentiation, or in vivo differentiation (Figure 2). 
a
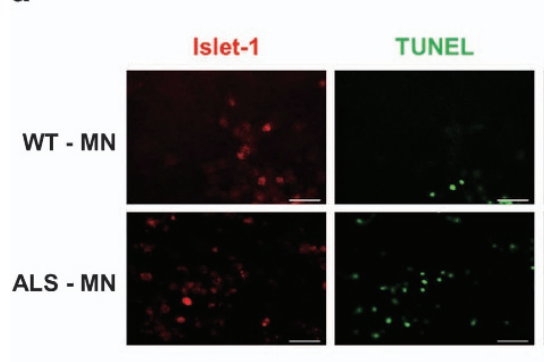

C

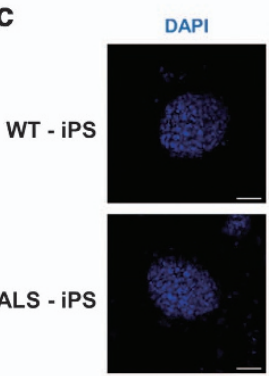

TUNEL

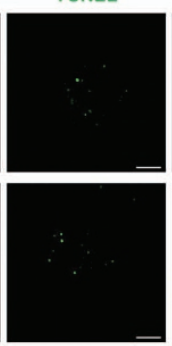

Merged

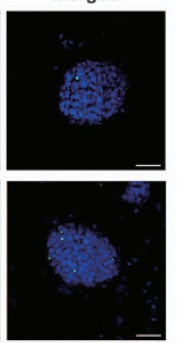

Merged

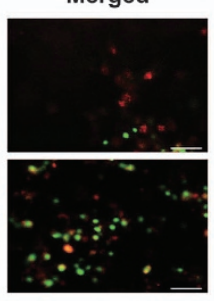

b

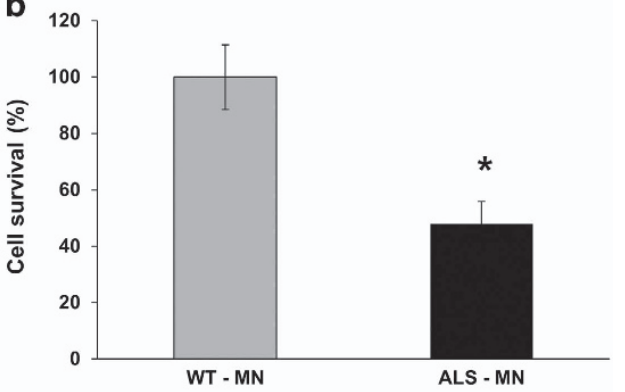

Figure 5 ALS-motor neurons were more TUNEL positive than WT-motor neurons. (a) Cell death was measured by TUNEL assays in WTMNs and ALS-MNs. Islet-1 (red) represents motor neurons and TUNEL (green) represents cell death. Scale bar, $200 \mu \mathrm{m}$. (b) TUNEL assays show that cell survival of ALS-MNs (47.9\%) was smaller than cell survival in WT-MNs $(P<0.005)$. The results reflect six independent experiments. Error bar reflects s.d. (c) Cell death was measured by TUNEL assays in WT-iPS and ALS-iPS colonies. TUNEL (green) represents cell death. Scale bar, $50 \mu \mathrm{m}$. (d) TUNEL assays in WT-iPS and ALS-iPS colonies. ( $n=20$ (WT), 15 (ALS)) Error bar reflects s.d.

Therefore, we can assume that the pathological symptoms of ALS would not occur in the embryonic stage.

To elucidate the differences in the developmental stage of the ALS model, we used a five-stage method to differentiate motor neuron cells from the miPS cells (Figure 3). We divided motor neuron differentiation into the iPS stage, the neural precursor stage, and the motor neuron stage, similar to that of embryonic development in vivo. Studies of motor neuron differentiation showed that the mutant SOD1 gene did not affect the rate of differentiation of neural precursor cells into motor neurons derived from ALS-iPS.

The major pathology of ALS includes motor neuronal cell death, a decrease in motor neuron neurites, and the presence of mutant SOD1 aggregates. $^{23-25}$ In previous studies, these ALS pathologies were presented in G93A SOD1 transgenic mice, an ALS model. Therefore, we investigated WT- and ALS-motor neurons derived from the miPS cells to confirm whether these pathologies were reproducible (Figure 4). In this study, the length of ALS-MN neurites decreased more than the neurites from the WT-MNs, and mutant SOD1 aggregates were present in ALS-MNs but not in WT-MNs. Moreover, the cell survival rate of ALS-MNs also decreased (Figure 5). In particular, the difference in the cell death rate between WT and ALS was present in the motor neuron stage. Thus, ALS-motor neurons derived from the miPS cells recapitulated various pathologies found in the G93A transgenic mouse model of ALS.

Although G93A mutant SOD1 retains native enzyme activity, oxidative stress may accelerate the aggregation of G93A SOD $1 .{ }^{26}$ Since SOD1 is an anti-oxidant enzyme and converts superoxide to hydrogen peroxide, G93A SOD1 suffers from oxidative damage more easily than other proteins. ${ }^{6}$ Moreover, oxidation of SOD1 is caused by hydrogen peroxide, and mutant SOD1 has a more increased affinity for hydrogen peroxide than wild-type SOD1. ${ }^{27,28}$ Thus, oxidized G93A SOD1 proteins have conformational changes that are strongly prone to becoming insoluble aggregates. ${ }^{29}$ These insoluble aggregates may be the reason for the fragmented neural fibers observed in the differentiated motor neurons from ALS-iPS cells (Figure 4d).

In a previous study, ${ }^{30}$ researchers generated motor neurons from ALS transgenic mouse-derived miPS cells, but they did not use the miPS-derived motor neurons in further studies. In the present study, we carried out functional studies of the properties of miPS-derived motor neurons, showing that miPS cells recapitulate various pathologies in the ALS model. Therefore, ALS-MNs derived from miPS cells could provide an effective model for drug screening or experimental subjects, although our study was limited to molecular biological research. Further studies should investigate genetic changes of iPS cells and motor neurons. Our study results imply that motor neurons derived from miPS cells could have many advantages for the study of the molecular pathogenesis of other neurodegenerative diseases.

\section{CONFLICT OF INTEREST}

The authors declare no conflict of interest.

\section{ACKNOWLEDGEMENTS}

This work was supported by the Korea Healthcare Technology R\&D Project, Ministry for Health, Welfare \& Family Affairs, Republic of 
Korea (no. A120340), a grant from the National R\&D Program for Cancer Control, the Ministry of Health \& Welfare, Republic of Korea (no. 1320010), the National Research Foundation of Korea Grants, the Ministry of Science, ICT and Future Planning, Republic of Korea (NRF-2015R1A4A1041919) and the National Research Foundation of Korea (NRF) grant (MEST) (NRF-2015R1A2A2A01003516).

1 Mulder DW. Clinical limits of amyotrophic lateral sclerosis. Adv Neurol 1982; 36: 15-22.

2 Ferraiuolo L, Kirby J, Grierson AJ, Sendtner M, Shaw PJ. Molecular pathways of motor neuron injury in amyotrophic lateral sclerosis. Nat Rev Neurol 2011; 7: 616-630.

3 Andersen PM, Sims KB, Xin WW, Kiely R, O'Neill G, Ravits J et al. Sixteen novel mutations in the $\mathrm{Cu} / \mathrm{Zn}$ superoxide dismutase gene in amyotrophic lateral sclerosis: a decade of discoveries, defects and disputes. Amyotroph Lateral Scler Other Motor Neuron Disord 2003; 4: 62-73.

4 Reaume AG, Elliott JL, Hoffman EK, Kowall NW, Ferrante RJ, Siwek DF et al. Motor neurons in $\mathrm{Cu} / \mathrm{Zn}$ superoxide dismutase-deficient mice develop normally but exhibit enhanced cell death after axonal injury. Nat Genet 1996; 13: 43-47.

5 Rosen DR. Mutations in Cu/Zn superoxide dismutase gene are associated with familial amyotrophic lateral sclerosis. Nature 1993; 364: 362.

6 Andrus PK, Fleck TJ, Gurney ME, Hall ED. Protein oxidative damage in a transgenic mouse model of familial amyotrophic lateral sclerosis. J Neurochem 1998; 71: 2041-2048.

7 Liu J, Lillo C, Jonsson PA, Vande Velde C, Ward CM, Miller TM et al. Toxicity of familial ALS-linked SOD1 mutants from selective recruitment to spinal mitochondria. Neuron 2004; 43: 5-17.

8 Nishitoh H, Kadowaki H, Nagai A, Maruyama T, Yokota T, Fukutomi H et al. ALS-linked mutant SOD1 induces ER stress- and ASK1-dependent motor neuron death by targeting Derlin-1. Genes Dev 2008; 22: 1451-1464.

9 Van Damme P, Dewil M, Robberecht W, Van Den Bosch L. Excitotoxicity and amyotrophic lateral sclerosis. Neurodegener Dis 2005; 2: 147-159.

10 Cheroni C, Marino M, Tortarolo M, Veglianese P, De Biasi S, Fontana E et al. Functional alterations of the ubiquitin-proteasome system in motor neurons of a mouse model of familial amyotrophic lateral sclerosis. Hum Mol Genet 2009; 18: 82-96.

11 Bilsland LG, Sahai E, Kelly G, Golding M, Greensmith L, Schiavo G. Deficits in axonal transport precede ALS symptoms in vivo. Proc Natl Acad Sci USA 2010; 107: 20523-20528.

12 Kirby J, Halligan E, Baptista MJ, Allen S, Heath PR, Holden H et al. Mutant SOD1 alters the motor neuronal transcriptome: implications for familial ALS. Brain 2005; 128(Pt 7): 1686-1706.

13 Johnston JA, Dalton MJ, Gurney ME, Kopito RR. Formation of high molecular weight complexes of mutant $\mathrm{Cu}, \mathrm{Zn}$-superoxide dismutase in a mouse model for familial amyotrophic lateral sclerosis. Proc Natl Acad Sci USA 2000; 97: 12571-12576.

14 Gurney ME, Pu H, Chiu AY, Dal Canto MC, Polchow CY, Alexander DD et al. Motor neuron degeneration in mice that express a human $\mathrm{Cu}, \mathrm{Zn}$ superoxide dismutase mutation. Science 1994; 264: 1772-1775.

15 Turner BJ, Talbot K. Transgenics, toxicity and therapeutics in rodent models of mutant SOD1-mediated familial ALS. Prog Neurobiol 2008; 85: 94-134.

16 Takahashi K, Yamanaka S. Induction of pluripotent stem cells from mouse embryonic and adult fibroblast cultures by defined factors. Cell 2006; 126: 663-676.
17 Robinton DA, Daley GQ. The promise of induced pluripotent stem cells in research and therapy. Nature 2012; 481: 295-305.

18 Dimos JT, Rodolfa KT, Niakan KK, Weisenthal LM, Mitsumoto H, Chung W et al. Induced pluripotent stem cells generated from patients with ALS can be differentiated into motor neurons. Science 2008; 321: 1218-1221.

19 Bilican B, Serio A, Barmada SJ, Nishimura AL, Sullivan GJ, Carrasco M et al. Mutant induced pluripotent stem cell lines recapitulate aspects of TDP-43 proteinopathies and reveal cell-specific vulnerability. Proc Natl Acad Sci USA 2012; 109: 5803-5808.

20 Takahashi K, Okita K, Nakagawa M, Yamanaka S. Induction of pluripotent stem cells from fibroblast cultures. Nat Protoc 2007; 2: 3081-3089.

21 Park HS, Hwang I, Choi KA, Jeong H, Lee JY, Hong S. Generation of induced pluripotent stem cells without genetic defects by small molecules. Biomaterials 2015; 39: 47-58.

22 Okabe S, Forsberg-Nilsson K, Spiro AC, Segal M, McKay RD. Development of neuronal precursor cells and functional postmitotic neurons from embryonic stem cells in vitro. Mech Dev 1996; 59 : 89-102.

23 Ince PG, Tomkins J, Slade JY, Thatcher NM, Shaw PJ. Amyotrophic lateral sclerosis associated with genetic abnormalities in the gene encoding $\mathrm{Cu} / \mathrm{Zn}$ superoxide dismutase: molecular pathology of five new cases, and comparison with previous reports and 73 sporadic cases of ALS. J Neuropathol Exp Neurol 1998; 57: 895-904.

24 Karumbayaram S, Kelly TK, Paucar AA, Roe AJ, Umbach JA, Charles A et al. Human embryonic stem cell-derived motor neurons expressing SOD1 mutants exhibit typical signs of motor neuron degeneration linked to ALS. Dis Model Mech 2009; 2: 189-195.

25 Chen H, Qian K, Du Z, Cao J, Petersen A, Liu H et al. Modeling ALS with iPSCs reveals that mutant SOD1 misregulates neurofilament balance in motor neurons. Cell Stem Cell 2014; 14: 796-809.

26 Rakhit R, Cunningham P, Furtos-Matei A, Dahan S, Qi XF, Crow JP et al. Oxidation-induced misfolding and aggregation of superoxide dismutase and its implications for amyotrophic lateral sclerosis. J Biol Chem 2002; 277: 47551-47556.

27 Yim HS, Kang JH, Chock PB, Stadtman ER, Yim MB. A familial amyotrophic lateral sclerosis-associated A4V Cu, Zn-superoxide dismutase mutant has a lower $\mathrm{Km}$ for hydrogen peroxide. Correlation between clinical severity and the Km value. J Biol Chem 1997; 272 8861-8863.

28 Wiedau-Pazos M, Goto JJ, Rabizadeh S, Gralla EB, Roe JA, Lee MK et al. Altered reactivity of superoxide dismutase in familial amyotrophic lateral sclerosis. Science 1996; 271: 515-518.

29 Valentine JS, Hart PJ. Misfolded CuZnSOD and amyotrophic lateral sclerosis. Proc Natl Acad Sci USA 2003; 100: 3617-3622.

30 Yao XL, Ye CH, Liu Q, Wan JB, Zhen J, Xiang AP et al. Motoneuron differentiation of induced pluripotent stem cells from SOD1G93A mice. PLOS ONE 2013; 8: e64720.

(1)(2) This work is licensed under a Creative Commons Attribution-NonCommercial-ShareAlike 4.0 International License. The images or other third party material in this article are included in the article's Creative Commons license, unless indicated otherwise in the credit line; if the material is not included under the Creative Commons license, users will need to obtain permission from the license holder to reproduce the material. To view a copy of this license, visit http:// creativecommons.org/licenses/by-nc-sa/4.0/ 